\title{
Bisphosphonate-related osteonecrosis of the jaw: specificities
}

\author{
Siri Paulo, ${ }^{1}$ Ana Margarida Abrantes, ${ }^{1}$ Mafalda Laranjo, ${ }^{1}$ Lina Carvalho, ${ }^{1}$ Arménio Serra, ${ }^{2}$ \\ Maria Filomena Botelho, ${ }^{1}$ Manuel Marques Ferreira' \\ ${ }^{1}$ Faculty of Medicine, Coimbra University; ${ }^{2}$ Faculty of Science and Technology, Coimbra University,
Portugal
}

\begin{abstract}
Bisphosphonate-related osteonecrosis of the jaws (BRONJ) is a severe complication that has recently emerged in patients treated with intravenous bisphosphonates for malignant diseases. This complication usually presents after a minor local trauma during a dental treatment. Several etiopathogenic mechanisms of this pathological condition have been proposed, but no model can explain all morphological changes observed at the macroscopic and microscopic level. BRONJ is likely to be related to direct toxicity in the bone and soft tissue cells, due to nitrogen-containing bisphosphonates. This review elucidates the clinical indications and mechanism of action of bisphosphonates, reports some clinical diagnostic criteria for BRONJ, describe the histopathological criteria for BRONJ diagnosis, the potential triggering pathways and the available treatment strategies.
\end{abstract}

\section{Introduction}

The use of bisphosphonates (BPs) has dramatically increased over the last few years. They offer substantial clinical benefits when an imbalance between osteoblast-mediated bone deposition and osteoclast-mediated bone resorption underlies a pathology. ${ }^{1}$ The main benefit of this therapy is the prevention and treatment of cancer-related bone complications, a consequence of excessive bone metabolism, associated with bone metastases. ${ }^{2}$ The high affinity of bisphosphonates with bone compared to other tissues allows them to achieve a high concentration throughout the skeleton, mainly in the jaws. ${ }^{1}$ Additionally, after an invasive dental procedure causing a pH decrease, BPs accumulate in concentrations which are directly toxic for the oral epithelium. Bisphosphonates-related osteonecrosis of the jaws

Correspondence: Siri Paulo, Faculty of Medicine, Coimbra University, Portugal.

E-mail: sirivpaulo@gmail.com

Key words: bone, bisphosphonate, osteonecrosis, jaw, soft tissues.

Received for publication: 13 May 2014.

Revision received: 1 August 2014.

Accepted for publication: 4 August 2014.

This work is licensed under a Creative Commons Attribution NonCommercial 3.0 License (CC BY-NC 3.0).

CC Copyright S. Paulo et al., 2014

Licensee PAGEPress, Italy

Oncology Reviews 2014; 8:254

doi:10.4081/oncol.2014.254
(BRONJ) is diagnosed, if the oral wound remains with an exposed necrotic bone for at least eight weeks. BRONJ is an increasingly present adverse effect of a therapy that is intended to safeguard the patient's quality of life. Further knowledge of BPs mechanism of action, BRONJ diagnosis and its pathophysiology will provide a better understanding of the importance of prevention and treatment options.

\section{Bisphosphonates}

\section{Basis of their clinical indications}

Biochemical interactions between the bone microenvironment and cancer cells create a vicious cycle that promotes bone destruction and tumor growth. ${ }^{2}$ In patients with advanced malignancy, the primary tumor invades the bone and causes skeletal metastases. The metastasis disrupts the normal bone homeostasis by expressing growth factors and cytokines that accelerate osteoclast activity. Bone metabolism is excessive and leads to skeletal morbidity with bone pain, hypercalcemia, pathologic fractures and spinal cord compression..$^{2-4}$

Regulation of the normal bone homeostasis occurs via the RANK/RANKL/OPG pathway, in which the osteoblast lineage regulates the differentiation and activity of osteoclasts. ${ }^{5}$ This pathway involves three major components: receptor activator of nuclear factor B (RANK), which is expressed on the surface of osteoclast precursor cells; RANK ligand (RANKL), a polypeptide found on the surface of osteoblast cells and proteolytically released as a soluble form; and osteoprotegrin (OPG), which is a receptor produced by osteoblasts. ${ }^{5,6}$ Because differentiation and activation of osteoclast precursor cells into mature osteoclasts requires binding of RANKL to RANK, excessive bone resorption is prevented by OPG which binds to RANKL and avoids the interaction between RANKL and RANK ${ }^{5,6}$ This interaction is lost when cancer cells invade the bone microenvironment and induce excessive osteolysis, due to the increase of parathyroid hormone-related protein, which stimulates RANKL production by osteoblasts, and to the decrease of osteoblastic OPG. ${ }^{6,7}$

The pharmacological effect of BPs is related to their stable bond with the inorganic bone components and their biochemical effects on cells, predominantly osteoclasts. ${ }^{3,7}$ Intravenous BPs are frequently administered to patients with osteolytic metastases and at high risk of significant morbidity, as frequently occurs in patients with advanced solid tumors, particularly breast and prostate cancers, or multiple myeloma. ${ }^{2}$ The reduction in the activity and number of osteoclasts decreases bone resorption, preventing, reducing and delaying cancer skeletal complications. ${ }^{2,4}$

BPs are also the major class of drugs used for the treatment of osteoporosis and other diseases characterized by excessive bone resorption, whereby more bone is destroyed than formed, thus leading to a negative balance. Besides reducing bone resorption through their action in osteoclasts, BPs also have a selective uptake and long-term presence in the skeleton. ${ }^{8}$ 


\section{Mechanism of action}

BPs are analogues of inorganic pyrophosphate (PPi), an endogenous regulator of bone mineralization, capable of inhibiting calcification. PPi (Figure 1) is a source of inorganic phosphate (Pi), which sustains the formation of hydroxyapatite when hydrolyzed, but it is also a potent inhibitor of apatite mineral deposition and growth. ${ }^{1,9}$

Like their natural analogue, BPs have a very high affinity with bone minerals, because they bind to hydroxyapatite crystals. They are preferentially incorporated into sites of active bone remodeling, as commonly occurs in conditions characterized by an accelerated skeletal turnover. ${ }^{7}$

Instead of the central oxygen atom in the core structure (P-0-P), bisphosphonates contain a central non-hydrolysable carbon (P-C-P), even though the phosphate groups adjacent to this central carbon are maintained. They also contain two side chains, Radical 1 and Radical 2, which are attached to the carbon atom. Nearly all bisphosphonates used in clinical practice have a hydroxyl group attached to the central carbon in the position of Radical 1 (Figure 2).,7

The adjacent phosphate groups provide the high affinity of bisphosphonates with bone hydroxyapatite crystals, whereas the hydroxyl group increases bisphosphonate's ability to bind calcium. Collectively, the phosphate and the hydroxyl groups create a tertiary interaction between the bisphosphonates and the bone matrix, making them particular specific for bones. . $^{1,10,11}$

The Radical 2 chain determines the anti-resorptive potency and its efficiency. Changes in this chain give each bisphosphonate their own chemical, physicochemical and biological characteristics. ${ }^{11,12}$

The high affinity of bisphosphonates with bone compared to other tissues allows them to achieve a high concentration throughout the skeleton. They are preferentially incorporated into sites of active bone remodeling. Bisphosphonates that do not enter the skeleton are rapidly cleared from the blood stream by renal excretion. ${ }^{1}$

The bisphosphonates anti-resorptive potency (Table 1) has increased from one generation to the next with the elongation of the Radical 2 side chain, the incorporation of an amino group and lately the incorporation of a tertiary amino group in the third generation. This has increased its potency, which is now 10,000 times greater than that of first generation of non-nitrogen-containing bisphosphonates. ${ }^{1,11,12}$

The nitrogen-containing bisphosphonates (nBP) inhibit the activity of farnesyl pyrophosphate synthase (FPPS), a key regulatory enzyme in the mevalonate pathway, which is crucial in the production of cholesterol, other sterols and prenylated proteins (Figure 3). The lack of this production interferes with the regulation of core cellular activities including stress fiber assembly, membrane ruffling and protein trafficking, including the cytoskeleton of the cells, thus resulting into apoptosis. ${ }^{1}$ Although farnesyl pyrophosphate synthase is ubiquitously expressed in mammalian cells, cellular apoptosis induced by nitrogen-<smiles>O=P(O)(O)OP(=O)(O)O</smiles>

Figure 1. Pyrophosphate chemical structure.<smiles>[R1]C([R2])(P(=O)(O)O)P(=O)(O)O</smiles>

Figure 2. Generic bisphosphonates chemical structure.

Mevalonate pathway
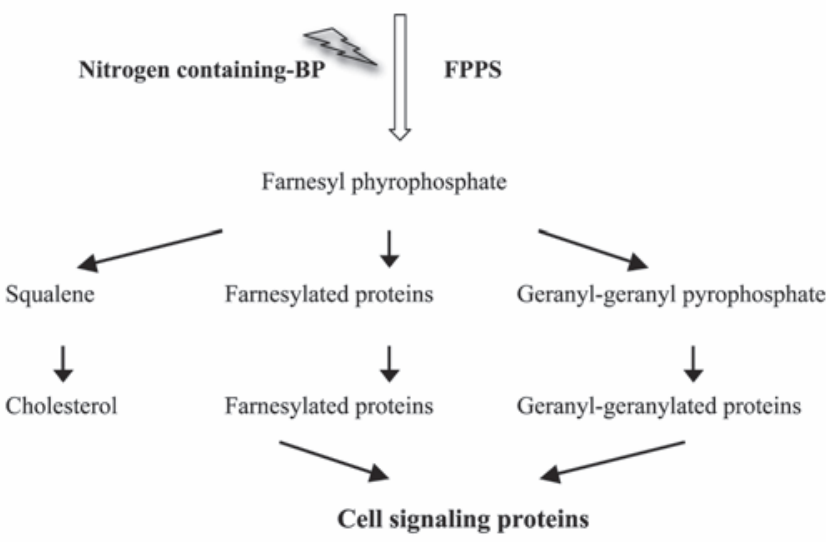

Figure 3. Mevalonate pathway - a metabolic pathway which plays a key role in multiple cellular processes. BP, bisphosphonate; FPPS, farnesyl pyrophosphate synthase.

Table 1. Non-nitrogen/nitrogen-containing bisphosphonates grouped according to their potency. Structures of the R1 and R2 side chains.

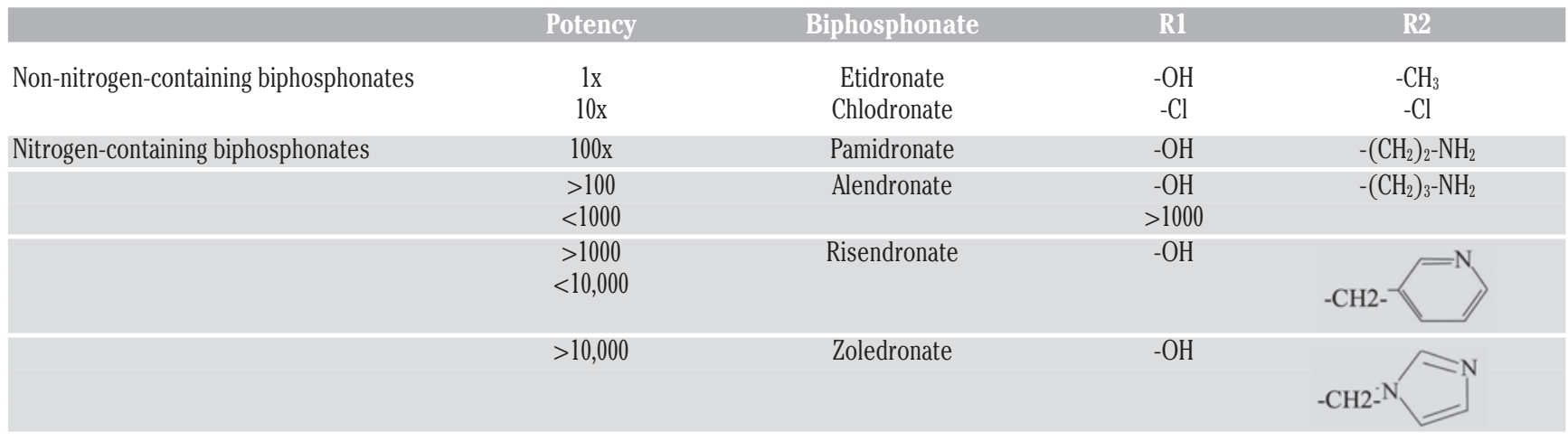


containing bisphosphonates occur mainly in osteoclasts. This demonstrates the ability of bisphosphonates to adhere to the bone before osteoclast endocytosis, during bone mineral dissolution. ${ }^{1,10}$

\section{Bisphosphonates-related osteonecrosis of the jaws}

In 2009 the American Association of Oral and Maxillofacial Surgeons (AAOMS $)^{13}$ stated that the diagnosis of BRONJ is primarily determined by the presence of exposed necrotic bone in the oral cavity during an eight-week period, a history of current or previous bisphosphonate intake, and the absence of radiation to the jaws. In addition to bone exposure, common features are pain, soft tissue swelling, ulceration, suppuration, and sinus tracts. ${ }^{14}$

Signs and symptoms which may occur before the development of clinically detectable osteonecrosis include pain, mucosal swelling, erythema and ulceration. In most cases these complications are triggered in sites of previous dental extraction..$^{13}$

The diagnosis of BRONJ relies on bone exposure, histological evidence of empty lacunae, absence of any neoplasm and a history of BPs use. $^{15}$

Early stages of BRONJ are difficult to detect via conventional radiography, which will not detect alterations until almost $50 \%$ of the bone is demineralized. 5,14

99mTc-methylene diphosphonate 3-phase bone scans have a limited specificity, which is increased by hybrid single photon emission computed tomography/computerized tomography. Bone scans may be an early indicator demonstrating tracer uptake even before $\mathrm{x}$-rays or clinical evidence. ${ }^{15}$ Although radionucleotide bone scans have a limited specificity, they are useful to detect changes in bone vascularity, and may be helpful to detect vascular changes at an early stage of BRONJ. Therefore, more than one imaging technique should be used, in order to gather both morphological and functional information.

These patients usually complain of pain and exposed necrotic bone surrounding inflammation. The management of these cases is difficult. Most of the available guidelines recommend a conservative treatment to eliminate pain, control infection and minimize the progression of bone necrosis. A systemic antibiotic treatment and an antimicrobial mouthwash are used in patients with asymptomatic exposed bone. A conservative palliative approach is recommended in patients with symptomatic exposed bone, because debridement is usually unsuccessful and can potentially expose healthy bone. ${ }^{14}$

The potency of BPs and the duration of treatment and dento-alveolar surgery are the main risk factors for BRONJ. ${ }^{13,16}$ A clinical staging system updated in the 2009 AAOMS guidelines is a useful tool to classify patients with BRONJ. It includes some treatment guidelines and explains how to collect data to assess prognosis and treatment outcome. $^{13}$

Stage 0 includes patients with no clinical evidence of necrotic bone, but with nonspecific clinical signs and symptoms, such as bone pain or osteosclerosis, that can be precursors of BRONJ development. These patients may require antibiotics and, if they have dental caries and periodontal disease, also a conservative treatment.,13,16

Patients with BRONJ in Stage 1 present exposed bone without soft tissue inflammation around the bone, which is usually asymptomatic and only requires periodic antibacterial oral rinses and close clinical follow up (Figures 4-6). If areas of exposed bone irritate the surrounding soft tissue, surface regularization could be helpful. ${ }^{4,13,16}$ Patients with BRONJ in Stage 2 have painful exposed necrotic bone and adjacent soft tissue inflammatory swelling. In most cases, penicillin is recommended. Necrotized tissue should be removed superficially in order to prevent secondary bone infection. ${ }^{4,13,16}$

In patients with BRONJ in Stage 3 the signs and symptoms described in Stage 2 are associated with pain and radiographic evidence of osteolysis, extra-oral fistulization or extensive sinusitis, typically refractory to antibiotics. At this stage, aggressive surgical management is

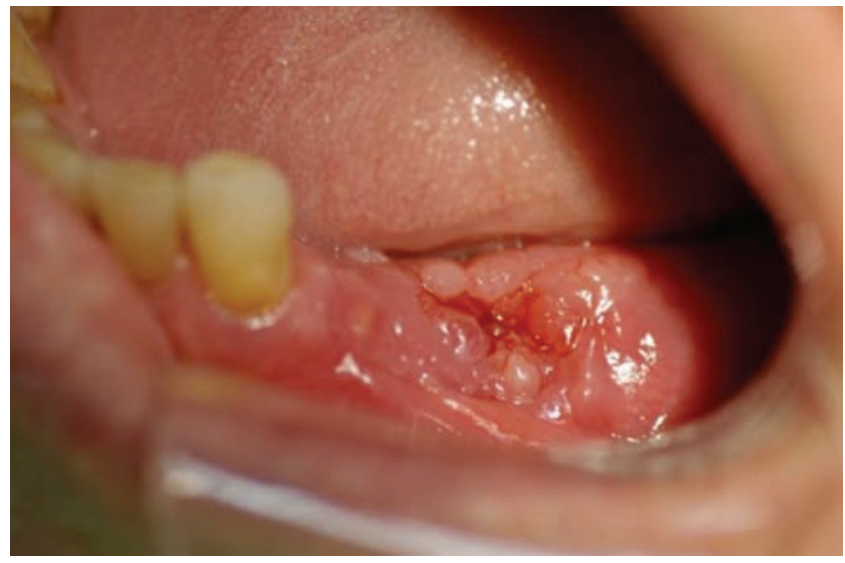

Figure 4. Patient with bisphosphonates-related osteonecrosis of the jaws in Stage 1.

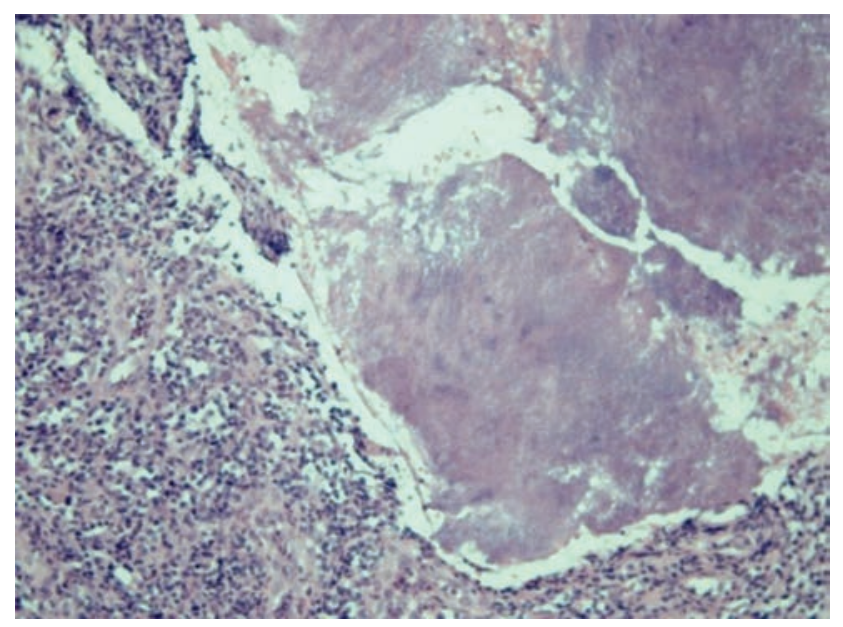

Figure 5. Histology (Grocott Stain x200 - Actinomyces).

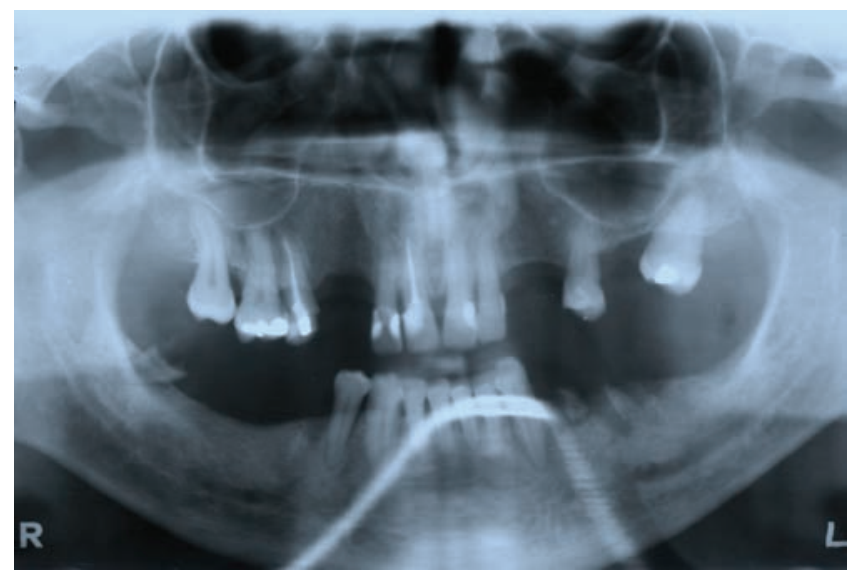

Figure 6. Panoramic radiography. 
required, so that relief with resolution of acute infection and pain can be ensured. $4,13,16$

Early diagnosis, timely treatment and therapeutic success during early stages of BRONJ are of crucial importance. No effective treatment is available for BRONJ which can lead to recurrent episodes of pain, swelling, infection and poorer quality of life, despite the treatment with bisphosphonates is be intended to improve it.

Nevertheless, various forms of osteonecrosis of the jaws (ONJ) have been described over the last 160 years, and a number of causes have been suggested in the literature. Evidence in the medical and dental literature reveals cases of $\mathrm{ONJ}$ associated with the use of drugs other than bisphosphonates, including denosumab, a human monoclonal antibody that inhibits the receptor activator of RANKL and antiangiogenic drugs tested in advanced cancer cases, such as bevacizumab, sunitinib, and possibly sorafenib. ${ }^{17}$

Among the existing cancer treatments, chemotherapy has the great inconvenience due to its lack of selectivity, since it acts on both tumor cells and rapidly multiplying normal cells, which can contribute to worsen BRONJ. ${ }^{18}$ Another therapy that may cause jaw osteonecrosis to occur earlier and be more severe is the use of steroids along with bisphosphonates. Therefore bisphosphonate- and steroid-related osteonecrosis of the jaws is more severe and unpredictable than BRONJ. 19

\section{Incidence}

With the introduction of nBPs, namely zoledronate (zoledronic acid), which has a powerful bone resorption inhibitor, the incidence of complications associated with intra-venous BPs has grown. ${ }^{16-23}$ The incidence of BRONJ after dental extractions range from $1 \%$ to $11 \%$ in breast cancer patients, $3 \%$ to $17 \%$ in multiple myeloma patients, and $3 \%$ to $18 \%$ in prostate cancer patients. ${ }^{20-22}$ Kühl et al. selected 23 studies and reported an incidence of BRONJ equal to $0-11.5 \%$ in therapies up to one year and $0-27.5 \%$ in therapies lasting from 1 to 4 years with the use of zoledronate. ${ }^{23}$

A survey was conducted to determine the prevalence of $\mathrm{ONJ}$ in a large community-based population with chronic oral bisphosphonate exposure. The recruitment and examination of the study cohort had well-defined criteria. Among the nearly 14,000 patients who had received chronic oral bisphosphonate therapy, 9 cases of $0 \mathrm{NJ}$ were identified with a minimum prevalence of $1(0.10 \%)$ in 952 respondents or $1(0.07 \%)$ in 1537 in the target population. ${ }^{24}$

\section{Why has the jaw an increased risk?}

The susceptibility the jaws to BRONJ may be due to several anatomical and physiological factors. Bisphosphonates tend to be highly concentrated in the jaws rather than in other skeletal sites, because they preferentially deposit in bones with high turnover rates, which are sites of significant remodeling. The forces of the masticatory system require a rapid bone turnover and can easily induce microfractures. ${ }^{25,26}$ Additionally, the jaw is separated from the oral environment by a thin epithelium, which can be easily traumatized and exposed to over 500 different species of microorganisms, thus displaying a high susceptibility to contamination and infection. ${ }^{27}$ Unlike other skeletal sites, after surgery or trauma, the wound can be continuously exposed to microorganisms. Under normal circumstances, an open bony wound in the presence of a normal oral microflora heals without complication, but in this cases oral microbes reaching the necrotic bone hinders the healing process. ${ }^{25}$

Another characteristic that distinguishes the jaw from other bones of the human skeleton is the type of ossification. The maxilla and mandible have an intramembranous ossification, unlike long bones and vertebrae, which have an endochondral ossification. ${ }^{28}$ The mandible is denser than any other bone in the human body. Its thickest section, where few vessels are present, is in the premolar and molar region, a site which is usually prone to BRONJ. Additionally, the jaw contains in general a fatty marrow and, in the presence of a hematopoietic environment, it lacks protection during the healing response of the bone. ${ }^{27,28}$

\section{Role of soft tissues in its pathophysiology}

Various mechanisms of BRONJ have been proposed, but its etiology is not yet fully understood. In order to explain the inadequate healing of wounds, which is the hallmark of BRONJ, some authors have focused on bone tissues, whereas others on soft tissues. To understand the basis of each theory, since most cases of BRONJ have, as trigger point, tooth extractions, it is necessary to consider the wound healing process. ${ }^{29,30}$

The blood clot forms within the first $24 \mathrm{~h}$ and is replaced by vascularized granulation tissue in following three days. ${ }^{29-31}$ By the seventh day, the provisional matrix is comprised of new blood vessels and collagen fibers. This is a vulnerable step, if the action of an agent inhibits osteoclasts, when in the adjacent bone marrow spaces osteoclasts should increase in number. ${ }^{29,30}$ By the $14^{\text {th }}$ day, large amounts of new woven bone are found, as well as newly formed blood vessels. Approximately one month after, this woven bone should remodel with an increased osteoclast activity and in the third month after tooth extraction it should be replaced by lamellar bone. This site only contains the bone marrow with lamellar bone after six months..$^{29-31}$

As a result, an impaired osteoclast function could hamper either the early remodeling of the old lamellar bone or the late remodeling of the new bone. Given these mechanisms, it is understandable that the primary lesion lies in the bone. Although, it is unclear why this lesion presents with a loss of soft tissue covering the maxillary bone as its primary clinical feature. ${ }^{32}$ This loss may be explained by the fact that BPs accumulate in bone at a high enough concentrations to be directly toxic for the oral epithelium. The lack of healing of the soft tissue lesions after an invasive dental procedure or trauma from dentures lead to secondary infection of the underlying bone, thus worsening the primary lesion. ${ }^{32,33}$

The ability of the different BPs to chelate $\mathrm{Ca}^{2+}$ decrease at lower $\mathrm{pH}$ levels due to the protonation of the BP phosphate groups. The protonated activation of the nitrogen-containing groups occurs at low $\mathrm{pH}$ levels $\left(\mathrm{NH}_{2}\right.$ to $\left.\mathrm{NH}_{3}\right)$, thus increasing the cytotoxicity in the local milieu. ${ }^{33}$ This hypothesis suggests that, with respect to infection, the microenvironment around active osteoclasts is highly acidic, inducing the release of BPs from the bone surface and creating high local concentrations. Not only does the local release of active BPs result in the inhibition of osteoclasts and impaired bone repair and healing, but it also induces direct cytotoxic effects on different cell types, so that cells in the immediate microenvironment, such as osteoblasts, endothelial cells, fibroblasts and keratinocytes, are be affected. ${ }^{32-34}$ The viability of these cells lines (monocytes, macrophages, periodontal ligament fibroblasts, oral keratinocytes, endothelial cells, osteoblasts and epithelial cells) is essential to wound healing. In this respect it has been demonstrated that these cell are negatively influenced by micro-molar concentrations. ${ }^{32-37}$ If BPs are constantly released from the underlining bone, the very thin mucosal layer is impaired. ${ }^{35}$ According to several authors, the cytotoxic effects of BPs on different cell types play a key role and $a^{14,17,32-35}$ theory that is gaining importance is the explanation of the pathophysiology as an outside-in process, through which mucosal damage provides access to the underlying bone, maintaining bone infection and necrosis. ${ }^{36-38}$

\section{Treatment strategies}

The undefined pathophysiology of BRONJ is, in part, responsible for the lack of reliable treatment strategies. BRONJ seems resistant to management of the open wound through curettage of the exposed bone and simple closure of the mucosa. This procedure can actually even worsen 
the prognosis. ${ }^{39}$ Also surgical resection, suggested in patients with BRONJ in Stage 3, remains controversial. ${ }^{40}$

Various therapies with superior outcome were presented and included prospective therapy, hyperbaric oxygen, parathyroid hormone and coverage of different flaps after surgery.

As mentioned above, nBPs inhibit FPPS in the mevalonate pathway. The decreased synthesis of geranylgeranyl-diphosphate affects vesicular transport, promote apoptosis of osteoclasts and undermines bone resorption, thus predisposing patients to the development of BRONJ. ${ }^{41}$ Ziebart et al. suggested that if a molecule of farnesyl pyrophosphate or geranylgeranyl pyrophosphate was supplied into the nBP-treated osteoclasts, the inhibitory effects of nBPs on osteoclasts could be compensated. ${ }^{41,42}$ These authors found that the addition of geranylgeranyl pyrophosphate restored the motility and RANK expression of the cultured osteoclast precursors, which were inhibited in the presence of zoledronate, implying the neutralization of nBPs effect. ${ }^{41}$ Such intermediate metabolites of the mevalonate pathway are expected to become drugs that can revive the osteoclasts in patients with BRONJ.

Nevertheless, osteoclast apoptosis can be suppressed by osteoclastogenic cytokines, tumor necrosis factor , macrophage colony-stimulating factor, RANKL, and interleukin- $6 .{ }^{43}$ As these are oxygen-sensitive, it was suggested that hyperbaric oxygen therapy (HBO) might influence their activity. ${ }^{43,44} \mathrm{HBO}$ may be promising, because it is expected to improve the hypoxia condition in the jaw and generate reactive oxygen species to stimulate the differentiation and activity of osteoclasts. ${ }^{45}$ Additionally, since hyperbaric oxygen is known to decrease edema and inflammation, enhance microbial killing, vasculogenesis and tissue repair in wounds, it can improve wound healing and pain scores when added to surgical and non-surgical treatment protocols. ${ }^{25}$

Teriparatide was also studied, because it maintains the N-terminal region of the parathyroid hormone (PTH) and recognizes its receptor (PTH receptor) expressed on the surface of osteoblasts. ${ }^{46}$ Teriparatide enhances the osteoblast function by inhibiting the apoptosis of osteoblasts and promoting the differentiation of osteoblast progenitors, the preosteoblasts. ${ }^{46}$ It can increase the metabolic activity of the osteoclast indirectly by directly affecting osteoblast function. ${ }^{47}$ Lau and Adachi highlighted that when PTH levels are continuously elevated and increase the serum calcium concentration, bones are severely degraded due to osteoclast activation. In contrast, an intermittent pulsed administration of PTH stimulates the differentiation and function of osteoblasts rather than osteoclasts, with anabolic effects on the bone. It can therefore be a valuable agent in the treatment of BRONJ.45 The last option is surgery with resection of the necrotic bone and a reliable soft tissue closure. A myofascial flap designed to cover the bony defect by exposing and detaching the mylohyoid muscle from mylohyoid line was proposed by Lemound et al. Twenty patients with BP-associated osteonecrosis of the lower jaw were successfully treated with this modified defect-covering method. ${ }^{48}$ Wilde $e t$ al. in a clinical study of 24 patients also obtain successful results, ${ }^{39}$ however Lorenzo et al. in a study of 8 patients suggested that only a well-vascularized flap must be used, as free flap, due to the structural instability of the mucosa in patients suffering from osteonecrosis. ${ }^{49}$

\section{Conclusions}

So far no conclusive and efficient therapeutic strategy has yet been developed for the treatment of BRONJ, therefore prevention should become a key priority. Dentists should assess the complete clinical history of their patients and avoid invasive dental treatments in subjects at risk of BRONJ. In high risk patients further risk stratification and detailed evaluation of the jaws may be achieved utilizing a bone scan. Additionally, oncologists should perform a baseline dental evaluation prior and during any treatment with intra-venous bisphosphonates.
The insufficient guidelines for the treatment of BRONJ and the growing use of BPs will amplify its impact on cancer patient's care. It is therefore imperative to gain further understanding of the pathophysiology of this disease and develop a local therapy that can prevent and/or reverse nBPs oral adverse effects. In relation to this review, the soft tissues and the wound healing capacity should attract particular attention and further investigation.

\section{References}

1. Drake MT, Clarke BL, Khosla S. Bisphosphonates: mechanism of action and role in clinical practice. Mayo Clin Proc 2008;83:1032-45.

2. Polascik TJ. Bisphosphonates in oncology: evidence for the prevention of skeletal events in patients with bone metastases. Drug Des Devel Ther 2009;21:27-40.

3. Mozzati M, Arata V, Gallesio G. Tooth extraction in patients on zoledronic acid therapy. Oral Oncol 2012;48:817-21.

4. Ruggiero SL, Drew SJ. Osteonecrosis of the jaws and bisphosphonate therapy. J Dental Res 2007;86:1013-21.

5. Pivonka P, Zimak J, Smith DW. Theoretical investigation of the role of the RANK-RANKL-OPG system in bone remodeling. $\mathrm{J}$ Theoret Biol 2010;262:306-16.

6. Gnant M, Clézardin P. Direct and indirect anticancer activity of bisphosphonates: a brief review of published literature. Cancer Treat Rev 2012;38:407-15.

7. Baron R, Ferrari S, Russel R. Denosumab and bisphosphonates: different mechanisms of action and effects. Bone 2011;48:677-92.

8. Russell R, Watts N, Ebetino F, Rogers M. Mechanisms of action of bisphosphonates: similarities and differences and their potential influence on clinical efficacy. Osteoporos Int 2008;19:733-59.

9. Thouverey C, Bechkoff G, Pikula S, Buchet R. Inorganic pyrophosphate as a regulator of hydroxyapatite or calcium pyrophosphate dihydrate mineral deposition by matrix vesicles. Osteoarthrit Cartilage 2009;17:64-72.

10. Cremers S, Papapoulos S. Pharmacology of bisphosphonates. Bone 2011;49:42.

11. Hewitt C, Farah CS. Bisphosphonate-related osteonecrosis of the jaws: a comprehensive review. J Oral Pathol Med 2007;36:319-28.

12. Papapoulos S. Bisphosphonates: how do they work? Best Pract Res Clin Endocrinol Metab 2008;22:831-47.

13. Ruggiero SL, Dodson TB, Assael LA, et al. American Association of Oral and Maxillofacial Surgeons position paper on bisphosphonaterelated osteonecrosis of the jaws: 2009 update. J Oral Maxillofac Surg 2009;67:2-12.

14. Nicolatou-Galitis 0, Papadopoulou E, Sarri T, et al. Osteonecrosis of the jaw in oncology patients treated with bisphosphonates: prospective experience of a dental oncology referral center. Oral Surg Oral Med Oral Pathol Oral Radiol Endod 2011;112:195-202.

15. Joshi JK, Kushner GM, Bhatt G, et al. Role of nuclear medicine imaging in recognizing different causes of osteonecrosis of the jaw. Clin Nucl Med 2013;38:40-3.

16. Coskun Benlidayi I, Guzel R. Oral bisphosphonate related osteonecrosis of the jaw: a challenging adverse effect. ISRN Rheumatol 2013;16:215034.

17. Yarom N, Elad S, Madrid C, et al. Osteonecrosis of the jaws induced by drugs other than bisphosphonates - a call to update terminology in light of new data. Oral Oncol 2010;46:e1.

18. Chaveli-Lopez B. Oral toxicity produced by chemotherapy: A systematic review. J Clin Exp Dent 2014;6:e81-90.

19. Chiu CT, Chiang WF, Chuang CY, Chang SW. Resolution of oral bisphosphonate and steroid-related osteonecrosis of the jaw-a serial case analysis. J Oral Maxillofac Surg 2010;68:1055-63. 
20. Hoff AO, Toth BB, Altundag K, et al. Frequency and risk factors associated with osteonecrosis of the jaw in cancer patients treated with intravenous bisphosphonates. J Bone Miner Res 2008;23:826-36.

21. Boonyapakorn T, Schirmer I, Reichart PA, et al. Bisphosphonateinduced osteonecrosis of the jaws: prospective study of 80 patients with multiple myeloma and other malignancies. Oral Oncol 2008;44:857-69.

22. Assaf AT, Smeets R, Riecke B, et al. Incidence of bisphosphonaterelated osteonecrosis of the jaw in consideration of primary diseases and concomitant therapies. Anticancer Res 2013;33:3917-24.

23. Kühl S, Walter C, Acham S, et al. Bisphosphonate-related osteonecrosis of the jaws - a review. Oral Oncol 2012;48:938-47.

24. Lo JC, 0'Ryan FS, Gordon NP, et al. Prevalence of ONJ after oral bisphosphonate exposure. J Oral Maxillofac Surg 2010;68:243-53.

25. Ikebe T. Pathophysiology of BRONJ: drug-related osteoclastic disease of the jaw. Oral Sci Int 2013;10:1-8.

26. Sarin J, DeRossi SS. Updates on bisphosphonates and potential pathobiology of bisphosphonate-induced jaw osteonecrosis. Oral Dis 2008;14:277-85.

27. Watts NB, Marciani RD. Osteonecrosis of the jaw. South Med J 2008;101:160-5.

28. Yu YY, Lieu S, Hu D, et al. Site specific effects of zoledronic acid during tibial and mandibular fracture repair. PLoS One 2012;7:e31771.

29. Szpaderska AM, Zuckerman JD, DiPietro LA. Differential injury responses in oral mucosal and cutaneous wounds. J Dent Res 2003;82:621-6.

30. Hämmerle CH, Araújo MG, Simion M. Osteology Consensus Group 2011. Evidence-based knowledge on the biology and treatment of extraction sockets. Clin Oral Implants Res 2012;23:80-2.

31. Evian CI, Rosenberg ES, Coslet JG, Corn H. The osteogenic activity of bone removed from healing extraction sockets in humans. J Periodontol 1982;53:81-5.

32. Reid IR, Bolland MJ, Grey AB. Is bisphosphonate-associated osteonecrosis of the jaw caused by soft tissue toxicity? Bone 2007;41:318-20.

33. Walter C, Klein MO, Pabst A, et al. Influence of bisphosphonates on endothelial cells, fibroblasts, and osteogenic cells. Clin Oral Investig 2010;14:35-41.

34. Scheper M, Chaisuparat R, Cullen K, Meiller T. A novel soft-tissue in vitro model for bisphosphonate-associated osteonecrosis. Fibrogenesis Tissue Repair 2010;1:3-6.

35. Pabst AM, Ziebart T, Koch FP, et al. The influence of bisphosphonates on viability, migration, and apoptosis of human oral keratinocytes - in vitro study. Clin Oral Investig 2012;16:87-93.
36. Cozin M, Pinker BM, Solemani K, et al. Novel therapy to reverse the cellular effects of bisphosphonates on primary human oral fibroblasts. J Oral Maxillofac Surg 2011;69:2564-78.

37. Agis $\mathrm{H}$, Blei J, Watzek G, Gruber R. Is zoledronate toxic to human periodontal fibroblasts? J Dent Res 2010;89:40-5.

38. Lesclous P, Abi Najm S, Carrel JP, et al. Bisphosphonate-associated osteonecrosis of the jaw: a key role of inflammation? Bone 2009;45:843-52.

39. Wilde F, Heufelder M, Winter K, et al. The role of surgical therapy in the management of intravenous bisphosphonates-related osteonecrosis of the jaw. Oral Surg Oral Med Oral Pathol Oral Radiol Endod 2011;111:153-63.

40. Carlson ER, Basile JD. The role of surgical resection in the management of bisphosphonate-related osteonecrosis of the jaws. $\mathrm{J}$ Oral Maxillofac Surg 2009;67:85-95.

41. Ziebart T, Koch F, Klein MO, et al. Geranylgeraniol - a new potential therapeutic approach to bisphosphonate associated osteonecrosis of the jaw. Oral Oncol 2011;47:195-201.

42. Kimachi K, Kajiya H, Nakayama S, et al. Zoledronic acid inhibits RANK expression and migration of osteoclast precursors during osteoclastogenesis. Naunyn-Schmiedebergs Arch Pharmacol 2011;383:297-308.

43. Ha H, Kwak HB, Lee SW, et al. Reactive oxygen species mediate RANK signaling in osteoclasts. Exp Cell Res 2004;301:119-27.

44. Freiberger JJ. Utility of hyperbaric oxygen in treatment of bisphosphonate- related osteonecrosis of the jaws. J Oral Maxillofac Surg 2009;67:96-106.

45. Lau AN, Adachi JD. Role of teriparatide in treatment of glucocorticoid-induced osteoporosis. Ther Clin Risk Manag 2010;6:497-503.

46. Subramanian G, Cohen HV, Quek SY. A model for the pathogenesis of bisphosphonate-associated osteonecrosis of the jaw and teriparatide's potential role in its resolution. Oral Surg Oral Med Oral Pathol Oral Radiol Endod 2011;112:744-53.

47. Harper RP, Fung E. Resolution of bisphosphonate-associated osteonecrosis of the mandible: possible application for intermittent low-dose parathyroid hormone [rhPTH(1-34)]. J Oral Maxillofac Surg 2007;65:573-80.

48. Lemound J, Eckardt A, Kokemüller H, et al. Bisphosphonate-associated osteonecrosis of the mandible: reliable soft tissue reconstruction using a local myofascial flap. Clin Oral Investig 2012;16:1143-52.

49. Lorenzo SD, Trapassi A, Corradino B, Cordova A. Histology of the oral mucosa in patients with BRONJ at III stage: a microscopic study proves the unsuitability of local mucosal flaps. J Clin Med Res $2013 ; 5: 22-5$. 